\title{
Azoxystrobin Reduces Oral Carcinogenesis by Suppressing Mitochondrial Complex III Activity and Inducing Apoptosis
}

This article was published in the following Dove Press journal: Cancer Management and Research

\author{
Hui Chen \\ Lingyu Li \\ Yunping Lu \\ Yajun Shen \\ Min Zhang (D) \\ Lihua Ge \\ Min Wang \\ Jing Yang \\ Zhenchuan Tian \\ Xiaofei Tang (D) \\ Division of Oral Pathology, Beijing \\ Institute of Dental Research, Beijing \\ Stomatological Hospital \& School of \\ Stomatology, Capital Medical University, \\ Beijing, People's Republic of China
}

Correspondence: Xiaofei Tang Division of Oral Pathology, Beijing Institute of Dental Research, Beijing Stomatological Hospital \& School of Stomatology, Capital Medical University, No. 4 Tiantan Xili, Dongcheng District, Beijing 100050, People's Republic of China Tel +86-|0-570993|I

Fax +86-10-570993I3

Email xftang10@ccmu.edu.cn
Purpose: The five-year survival rate of patients with oral cancer is approximately $50 \%$; thus, alternative drugs with higher efficacy are urgently required. Azoxystrobin (AZOX), a natural, novel methoxyacrylate fungicide isolated from mushrooms, has a broad-spectrum, with highly efficient bactericidal effect. However, studies on AZOX have focused on antifungal effects. Here, we explore the potential cancer-preventive effects of AZOX and the underlying mechanisms.

Materials and Methods: The effects of AZOX on oral carcinogenesis induced by 4-nitroquinoline-1-oxide (4NQO) were investigated in C57BL/6 mice. Cell proliferation and apoptosis were examined by Ki67 immunohistochemistry and TUNEL staining, respectively. The main organ coefficients of each group were calculated to evaluate the biosafety of AZOX. CCK8 and flow cytometry were used to detect the effects of AZOX on cell viability and apoptosis in oral cancer cell line CAL27 and SCC15 cells in vitro. Cell cycle, mitochondrial complex III activity, intercellular reactive oxygen species (ROS) level, mitochondrial ROS level, and mitochondrial membrane potential (MMP) were detected by flow cytometry in AZOX-treated CAL27 cells.

Results: AZOX significantly inhibited the occurrence of 4NQO-induced tongue cancer and delayed the progression of tongue precancerous lesions in mice. High-dose AZOX obviously inhibited cell viability and induced apoptosis in epithelial dysplastic and oral squamous cell carcinoma (OSCC) lesions in mouse tongue mucosa. AZOX was confirmed to have high biosafety. Similarly, in vitro cell viability was suppressed, and apoptosis was induced in AZOX-treated CAL27 and SCC15 cells. AZOX induced cell cycle arrest at the S phase. AZOX inhibited mitochondrial complex III activity, increased intracellular and mitochondrial ROS levels, and decreased MMP in CAL27 cells.

Conclusion: AZOX inhibited the development of oral cancer through specific inhibition of the activity of mitochondrial complex III, which led to ROS accumulation, and MMP decrease, ultimately inducing apoptosis. AZOX may be a novel agent for the prevention and treatment of OSCC.

Keywords: azoxystrobin, oral cancer, mitochondrial complex III, apoptosis

\section{Introduction}

Oral cancer is the sixth most common malignant tumor. More than 550,000 new cases and 300,000 deaths caused by oral squamous cell carcinoma (OSCC) are reported each year worldwide. ${ }^{1}$ Comprehensive OSCC treatment mainly includes surgery, radiotherapy, and chemotherapy. For most patients with advanced or distant metastases who cannot receive surgery and radiotherapy, chemotherapy is often 
used to inhibit tumor growth and reduce distant metastasis, thereby improving the quality of life and overall survival rate of the patients. ${ }^{2}$ However, only few drugs have been developed, and drug resistance and biological toxicity of drugs often lead to chemotherapy failure. Therefore, it is urgent to develop new anticancer drugs for the treatment of oral cancer.

Azoxystrobin (AZOX), also known as Amisida, is a natural insecticide from the family of strobilurins. Originally isolated from mushrooms, AZOX has the characteristics of broad-spectrum effects, high efficiency, and low toxicity. It has not been reported to be mutagenic, teratogenic, or carcinogenic. ${ }^{3,4}$ Studies have shown that AZOX is a mitochondrial complex III inhibitor that destroys ATP synthesis in fungal cells via inhibition of the mitochondrial respiratory chain. It also inhibits the genesis and growth of pathogenic spores and mycelial growth. ${ }^{5}$ AZOX $(0.20 \mathrm{mg} / \mathrm{L})$ reduces the mitochondrial complex III activity, increases the reactive oxygen species (ROS) level, and increases the superoxide dismutase activity in larval and adult zebrafish. ${ }^{6}$ We previously screened effective anticancer compounds from more than 70 natural plant compounds and discovered that AZOX obviously inhibited cell viability in oral cancer and precancerous cells. Recent studies have shown that AZOX inhibited the proliferation of KYSE-150 human esophageal cancer cells and induces apoptosis. ${ }^{7}$ However, the effects and mechanisms of AZOX in oral cancer are unknown.

We hypothesized that AZOX suppresses the development of oral cancer via the inhibition of mitochondrial complex III activity. To test this hypothesis, we used a 4-nitroquinoline1-oxide (4NQO)-induced mouse tongue cancer model and the oral cancer cell lines CAL27 and SCC15 to study the preventive effects of AZOX on oral carcinogenesis and investigate the underlying mechanisms by examining cell viability, apoptosis, and mitochondrial functions. This study may provide evidence for the development of a new drug for the treatment and prevention of oral cancer.

\section{Materials and Methods}

\section{Chemicals and Reagents}

AZOX was obtained from Sigma-Aldrich (MO, USA) and dissolved in dimethyl sulfoxide (DMSO) with $5 \mathrm{mg} / \mathrm{mL}$. For cell experiments, the solution was diluted with cell culture medium to $0,1.5,3,5,6$ and $12 \mu \mathrm{g} / \mathrm{mL}$ AZOX solutions. For animal experiments, the solution was diluted with corn oil to prepare high-dose $(25 \mathrm{mg} / \mathrm{kg})$ and low- dose $(5 \mathrm{mg} / \mathrm{kg})$ AZOX solutions. 4NQO was purchased from Sigma-Aldrich and dissolved in 1,2-propanediol. The solution was diluted with purified water to $50 \mu \mathrm{g} / \mathrm{mL}$ and kept in a dark bottle. Propidium iodide (PI) staining solution and Hoechst 33,342 were purchased from Thermo Fisher Scientific (MA, USA).

\section{Animal Experiments}

C57BL/6 mice (4 weeks old, 16-20 g) were purchased from Vital River Laboratory Animal Technology (Beijing, China). The experiments were conducted in accordance with the UK Animals (Scientific Procedures) Act and the Beijing Municipality on the Review of Welfare and Ethics of Laboratory Animals guidelines approved by the Beijing Municipality Administration Office of Laboratory Animals (BAOLA). Furthermore, the study was approved by the Animal Ethical and Welfare Committee of Beijing Stomatological Hospital (approval no. KQYY-201,804010). A total of 34 mice, 17 male and 17 female, were randomly divided into four groups: blank control $(n=4)$, high-dose AZOX $(n=10)$, low-dose AZOX $(n=10)$, and model control $(n=10)$. The sample sizes of the groups were chosen based on preliminary studies. All animals were in good physiological and immunological conditions and maintained at a controlled temperature under a $12 \mathrm{hrs}$ light/dark cycle. No treatment was given to the blank control group, whereas the remaining 30 mice were administered $50 \mu \mathrm{g} / \mathrm{mL} 4 \mathrm{NQO}$ water. From the 10th week, high-dose $(25 \mathrm{mg} / \mathrm{kg})$ and low-dose AZOX $(5 \mathrm{mg} / \mathrm{kg})$ were intragastrically administered every other day. The model control group was intragastrically administered the same dose of corn oil. The development of tongue lesions, overall health condition, and total body weight of the mice were examined weekly. Drinking of $4 \mathrm{NQO}$ water and intragastric administration were terminated at the end of 16 th and 24th weeks, respectively. When the mice had lost $25 \%$ of their original body weight, they were euthanized according to the BAOLA guidelines. The mice were euthanized by intraperitoneal injection of pentobarbitalphenytoin solution at the end of 24 th week, ${ }^{8}$ and their tongue, liver, kidney, and spleen were extracted, the flow chart of animal experiment is in Supplementary Figure 1. The tongue was cut longitudinally; one half of the tongue was stored at $-80{ }^{\circ} \mathrm{C}$ for molecular biological testing, whereas the other was fixed in $10 \%$ neutral formaldehyde for histological examination. 


\section{Hematoxylin and Eosin (H\&E) Staining}

Each tongue tissue was cut into $4 \mu \mathrm{m}$ thick sections continuously and the 1st, 5 th, and 10th sections were used for H\&E staining. The sections were observed using an Olympus BX61 microscope (Olympus, Tokyo, Japan). Histological changes in tongue mucosa and the degree of lesion were recorded according to the World Health Organization classification of head and neck tumors (4th Edition, 2017). The incidence of various lesions in each group was calculated.

\section{Immunohistochemistry}

After being routinely dewaxed and rehydrated, the paraffin-embedded slides were heated for $10 \mathrm{mins}$ in a pressure cooker containing $10 \mathrm{mM}$ citrate buffer $(\mathrm{pH}$ 6.0) to retrieve the antigens. Endogenous peroxidases were quenched by $3 \% \mathrm{H}_{2} \mathrm{O}_{2}$. After blocking, the sections were incubated with $\mathrm{Ki} 67$ working solution (Maxim Biotechnologies, China) overnight at $4{ }^{\circ} \mathrm{C}$ followed by secondary antibody incubation for 30 mins at room temperature. Freshly prepared DAB solution (Maxim Biotechnologies) was used for staining. The sections were counterstained with hematoxylin. Six fields were randomly selected in each slice for image acquisition under an Olympus BX61 microscope. The Image Pro Plus software (Media Cybernetics, Rockville, MD 20,852 USA) was used to calculate the positive cells.

\section{TUNEL Staining}

The sections were routinely dewaxed, rehydrated, and incubated with proteinase $\mathrm{K}(20 \mu \mathrm{g} / \mathrm{mL}$ in Tris/HCL $)$ at $37{ }^{\circ} \mathrm{C}$ for 15 mins. The sections were subsequently incubated with a TUNEL reaction solution (Roche, Germany) in light-free conditions at $37^{\circ} \mathrm{C}$ for 30 mins. Next, $50 \mu \mathrm{L}$ of converter-POD was added at $37{ }^{\circ} \mathrm{C}$ for 30 mins. After incubation with $\mathrm{DAB}$, the sections were counterstained with hematoxylin. Three fields were randomly selected in each slice for image acquisition under an Olympus BX61 microscope. The Image Pro Plus software was used to calculate the positive cells.

\section{Cell Culture}

Human OSCC CAL27 cells (CRL-20,957; American Type Culture Collection, ATCC) were cultured in high-glucose Dulbecco's modified Eagle's medium (DMEM-High glucose) containing 10\% fetal bovine serum (Gibco, USA), $100 \mathrm{U} / \mathrm{mL}$ penicillin, and streptomycin at $37{ }^{\circ} \mathrm{C}$ and $5 \%$
$\mathrm{CO}_{2}$. SCC15 human OSCC cells (CRL-1623; ATCC) were cultured in DMEM/F-12 medium containing $12.5 \%$ fetal bovine serum at $37{ }^{\circ} \mathrm{C}$ and $5 \% \mathrm{CO}_{2}$.

\section{Cell Viability Assay}

CAL27 and SCC15 cells were treated with AZOX at 1.5, 3,6 , and $12 \mu \mathrm{g} / \mathrm{mL}$ for 24,48 , and $72 \mathrm{hrs}$. The negative control cells were treated with the same concentration of DMSO for 24, 48, and $72 \mathrm{hrs}$. Cell viability was detected using CCK8 (Dojindo, Japan). The relative cell viability (\%) was presented as [OD] test wells/[OD] control wells $\times 100 \%$.

\section{Apoptosis Analysis}

After being treated with AZOX at 0 and $5 \mu \mathrm{g} / \mathrm{mL}$ for 24 and 48 hrs, CAL27 and SCC15 cells were incubated with Annexin V-fluorescein isothiocyanate for $15 \mathrm{mins}$ and PI for 5 mins in the dark. Early apoptotic (Annexin V+/PIcells) rates were analyzed by flow cytometry (BD, USA).

\section{Cell Cycle Detection}

CAL27 and SCC15 cells treated with 0 and $5 \mu \mathrm{g} / \mathrm{mL}$ of AZOX for 24 and $48 \mathrm{hrs}$ were fixed in $70 \%$ ethanol solution overnight at $4{ }^{\circ} \mathrm{C}$ and resuspended in PI working solution. Following incubation with RNase solution at $37^{\circ}$ $\mathrm{C}$ for 30 mins, the relative content of DNA in cells was determined by flow cytometry. The percentage of cells at each phase of the cell cycle was analyzed using the ModFit software (BD Bioscience, NJ, USA).

\section{Detection of Mitochondrial Complex III Activity}

After treatment with 0 and $5 \mu \mathrm{g} / \mathrm{mL}$ AZOX for $24 \mathrm{hrs}$, CAL27 cells were homogenized in an ice bath and centrifuged at $600 \times \mathrm{g}$ for $10 \mathrm{mins}$ at $4{ }^{\circ} \mathrm{C}$. The supernatant was transferred and centrifuged at $11,000 \times \mathrm{g}$ for $15 \mathrm{mins}$ at $4{ }^{\circ} \mathrm{C}$. The precipitate was ultrasonically disrupted in an ice bath. The detection solution was prepared using a micro mitochondrial respiratory chain complex III activity assay kit (Solarbio, China), according to the manufacturer's instructions. The absorbance values at $550 \mathrm{~nm}$ for the experimental and control groups at the beginning of the experiment were recorded as A1measurement and A1 control, respectively; the absorbance values at 2 mins were recorded as A2measurement and $\mathrm{A} 2$ control, respectively. $\Delta \mathrm{A}=$ (A2measurement - A1measurement) - (A2control Alcontrol). The samples were incubated with bicinchoninic 
acid working solution for $30 \mathrm{mins}$ at $37^{\circ} \mathrm{C}$. The absorbance at $562 \mathrm{~nm}$ was measured using a microplate reader, and the protein concentration was calculated using the standard curve. Mitochondrial complex III activity (U/mg prot) was calculated according to the kit instructions.

\section{Measurement of ROS Level}

CAL27 cells were treated with AZOX at $0,1.5,3,5$, and $6 \mu \mathrm{g} /$ $\mathrm{mL}$ for $24 \mathrm{hrs}$. For positive control cells, ROSup was added for 20 mins before conducting the experiment on the next day. The cells were suspended in $10 \mu \mathrm{mol} / \mathrm{L}$ of dihydroethidium and incubated at $37^{\circ} \mathrm{C}$ for $20 \mathrm{mins}$; the suspension was mixed once every 5 mins. Changes in fluorescence intensity were observed under an Olympus BX61 microscope (excitation wavelength: $535 \mathrm{~nm}$, emission wavelength: $610 \mathrm{~nm}$ ). Fluorescence intensity was measured by flow cytometry.

\section{Measurement of Intracellular Mitochondrial Superoxide}

CAL27 cells were treated with the same concentrations of AZOX as those mentioned in the "Measurement of ROS level" section. The cells, in a petri dish, were incubated with $2 \mathrm{~mL}$ of fluorescent probe staining solution $(5 \mu \mathrm{M})$ at $37^{\circ} \mathrm{C}$ for 10 mins in the dark, and changes in fluorescence intensity were observed under an Olympus BX61 microscope (excitation wavelength: $535 \mathrm{~nm}$, emission wavelength: $610 \mathrm{~nm}$ ). Cells were collected, and fluorescence intensity was detected by flow cytometry.

\section{Measurement of Mitochondrial Membrane Potential (MMP)}

CAL27 cells treated with AZOX as described in the "Measurement of ROS level" section were incubated with JC-1 staining solution (Biyuntian, China) at $37{ }^{\circ} \mathrm{C}$ for 20 mins. The cells treated with CCCP for 20 mins were used as a positive control. After the cells were washed with JC-1 staining buffer, the JC-1 monomer was detected at excitation and emission wavelengths of 490 and $530 \mathrm{~nm}$, respectively, whereas the JC-1 polymer was detected at excitation and emission wavelengths of 525 and $590 \mathrm{~nm}$, respectively. According to the manufacturer's instructions, in a high MMP condition, JC-1 accumulates in the matrix of the mitochondria to form polymeric JC1 aggregates, producing red fluorescence; in contrast, in a low MMP condition, JC-1 exists as its $\mathrm{JC} 1$-monomer, producing green fluorescence. The relative proportion of red and green fluorescence is a measure of mitochondrial depolarization.

\section{Statistical Analysis}

The experiments were conducted in triplicate. Statistical analysis was performed using the SPSS 17.0 statistical software (SPSS, Inc., Chicago, USA). Differences with $P$-values of less than 0.05 , as analyzed by variance analysis and an independent sample $t$-test, were considered statistically significant.

\section{Results}

\section{AZOX Reduces Tongue Cancer Development in Mice}

At the end of 10th weeks of drinking 4NQO, mouse body weight in the high- and low-dose AZOX groups decreased compared with that in the blank control group. Since there were no mice had lost $25 \%$ of their original body weight at the end of 24th weeks, no mice were sacrificed before the due date. There were no significant differences in the body weights of the high- and low-dose AZOX groups compared to the model control group at the end of 24th week ( $P>$ 0.05) (Figure 1A). Microscopically, epithelial hyperplasia, dysplasia (mild, moderate, and severe), and OSCC appeared in the tongue mucosa of mice in the model control, high-dose AZOX, and low-dose AZOX groups at the end of 24th week (Figure 1B). Compared with that in the model control group, the average number of precancerous and neoplastic tongue lesions decreased in the high-dose AZOX groups (Figure 1C). The incidence of tongue cancer was significantly lower in the high-dose AZOX group than that in the model control group $(P=0.009)$. The severity of tongue lesions was attenuated, and most lesions were at the epithelial mild-moderate dysplastic stage in the high-dose AZOX group, suggesting that AZOX delays or inhibits the development of tongue cancer.

In addition, the biosafety of AZOX was evaluated in mice. The main organ coefficients of each group were obtained. Compared with those in the model control group, there were no significant differences in liver, kidney, and spleen coefficients in both the high- and low-dose AZOX groups $(P>0.05)$ (Figure 1D), indicating that AZOX has higher biosafety.

\section{AZOX Inhibits Cell Proliferation in Mouse Tongue Mucosa}

Immunohistochemical staining showed that the rates of Ki67-positive cells in the dysplastic epithelial $(P=0.035)$ and OSCC $(P=0.032)$ tongue tissues were significantly lower in the high-dose AZOX group than in the model control group. Moreover, the rates of Ki67-positive cells in 
A

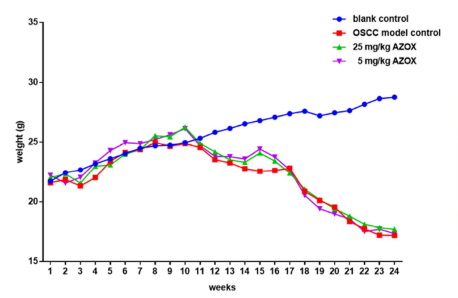

B
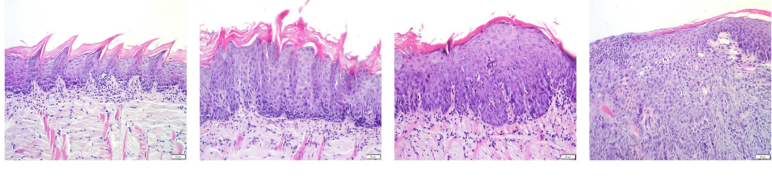

C

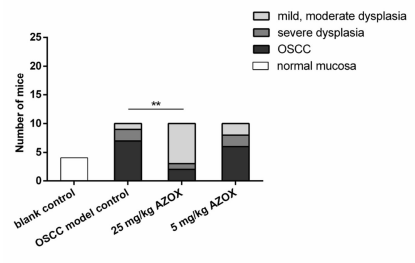

D
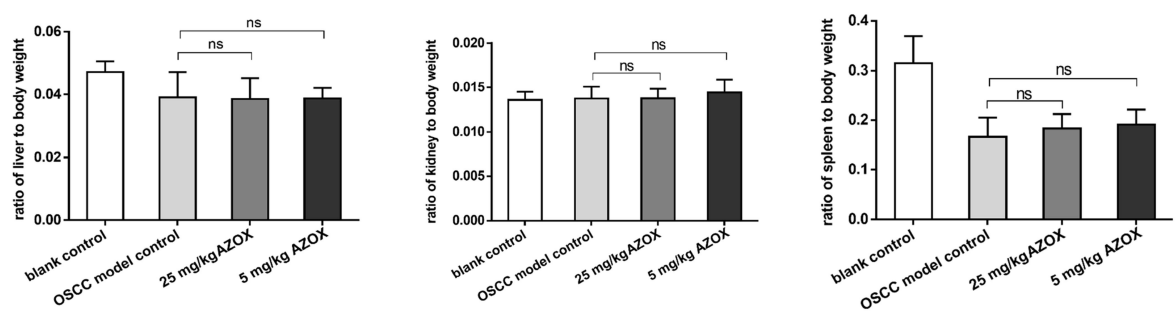

Figure I AZOX reduces tongue cancer development in mice.

Notes: (A) Body weight curves in the blank control, OSCC model, low-dose (5 mg/kg) AZOX, and high-dose (25 mg/kg) AZOX groups. (B) H\&E staining of mouse tongue mucosa. (C) All mice were sacrificed at the end of 24th week. Pathological diagnosis results of tongue mucosa in the blank control, OSCC model, low-dose AZOX, and highdose AZOX groups. In OSCC model control group, I of 10 mice had mild and moderate dysplasia lesions, 2 of 10 had severe dysplasia lesions, and 7 of 10 mice had OSCC lesions. In high-dose ( $25 \mathrm{mg} / \mathrm{kg}$ ) AZOX group, there were 7 of 10 mice had mild and moderate dysplasia lesions, I of 10 mice had severe dysplasia lesions, 2 of 10 mice had OSCC lesions. In low-dose (5 mg/kg) AZOX group, 2 of 10 mice had mild and moderate dysplasia lesions, 2 of 10 had severe dysplasia lesions, and 6 of 10 mice had OSCC lesions. (D) Main organ coefficients of each group. Data are presented as mean \pm standard deviation $(S D)(n=10)$. **P $<0.0$ I.

Abbreviations: OSCC, oral squamous cell carcinoma; AZOX, azoxystrobin; H\&E, hematoxylin and eosin; ns, no significance.

the dysplastic epithelial $(P=0.833)$ and $\operatorname{OSCC}(P=0.223)$ tongue tissues in the low-dose AZOX group were not significantly decreased (Figure 2), which suggests that high-dose AZOX obviously inhibits cell proliferation in dysplastic and OSCC tissues in mice.

\section{AZOX Induces Cell Apoptosis in Mouse Tongue Mucosa}

TUNEL assay showed that the apoptotic rate in the hyperplastic epithelia of the low- and high-dose AZOX groups was not significantly different from that in the epithelium of the model control group $(P=0.860$ and $P=0.110)$. In dysplastic tongue tissues, the apoptotic rate was significantly higher in the low- and high-dose AZOX groups than in the model control group $(P=0.026, P=0.017)$. In OSCC tissues, the apoptotic rate was significantly higher in the high-dose AZOX group than in the model control group $(P=0.035)$ (Figure 3$)$. These results suggest that AZOX induces apoptosis in dysplastic and OSCC tongue tissues in mice.

\section{AZOX Inhibits Cell Viability and Blocks the Cell Cycle in CAL27 and SCCI 5 Cells}

The effect of AZOX on the viability of OSCC cells was examined by CCK8 assay. As shown in Figure 4A, AZOX at
$1.5,3,6$, and $12 \mu \mathrm{g} / \mathrm{mL}$ significantly inhibited cell viability for 24, 48, and 72 hrs in CAL27 and SCC15 cells. The halfinhibitory concentration (IC50) values of AZOX in CAL27 cells treated for 24,48 , and $72 \mathrm{hrs}$ were $4.4,6.5$, and $5.9 \mu \mathrm{g} /$ $\mathrm{mL}$, respectively, and those of AZOX in SCC15 cells treated for 24,48 , and $72 \mathrm{hrs}$ were $7.82,7.51$, and $8.02 \mu \mathrm{g} / \mathrm{mL}$, respectively. These results suggest that AZOX inhibits CAL27 and SCC15 cell viability and that this effect of AZOX was more efficient in CAL27 cells than in SCC15 cells.

The effect of AZOX on the cell cycle was assessed in CAL27 and SCC 15 cells. First, $5 \mu \mathrm{g} / \mathrm{mL}$ AZOX was selected based on the IC50. The results showed that treatment of CAL27 cells with $5 \mu \mathrm{g} / \mathrm{mL}$ AZOX for $24 \mathrm{hrs}$ led to a significant cell cycle arrest in the S phase $(P=0.001)$ and a decrease in the number of cells in the $\mathrm{G} 0 / \mathrm{G} 1$ phase $(P=$ 0.002) (Figure 4B). SCC15 cells treated with $5 \mu \mathrm{g} / \mathrm{mL}$ AZOX for $48 \mathrm{hrs}$ were arrested in the $\mathrm{S}$ phase $(P=0.042)$ (Figure $4 C)$, which suggested that AZOX induces cell cycle arrest in the S phase in CAL27 and SCC15 cells and that CAL27 cells were more sensitive to AZOX treatment than $\mathrm{SCC} 15$ cells.

\section{AZOX Induces Apoptosis in CAL27 and SCCI5 Cells}

The early apoptotic rates in CAL27 cells treated with $5 \mu \mathrm{g} /$ $\mathrm{mL}$ AZOX for $24 \mathrm{hrs}$ increased compared with that in the 

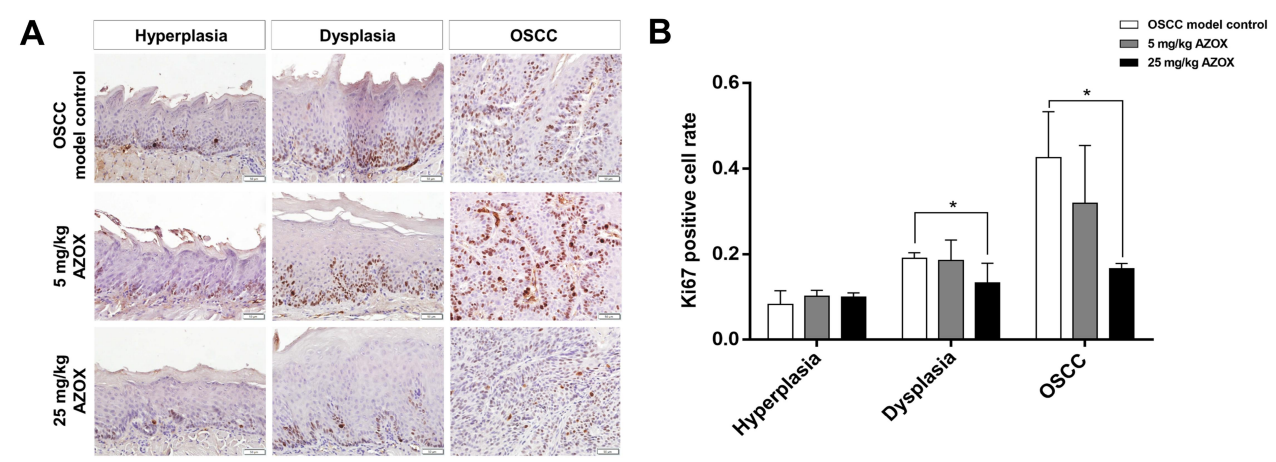

Figure 2 AZOX inhibits cell proliferation in mouse tongue mucosa.

Notes: (A) Immunohistochemical analysis of Ki67 staining in the OSCC model control, low-dose AZOX, and high-dose AZOX groups (magnification $\times 200$ ). (B) Cell proliferation rates in mouse tongue tissues in each group. Data are presented as mean \pm SDs. $* P<0.05$.

A

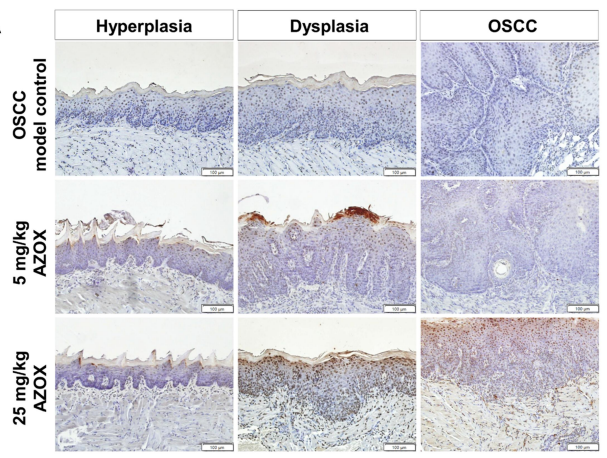

B

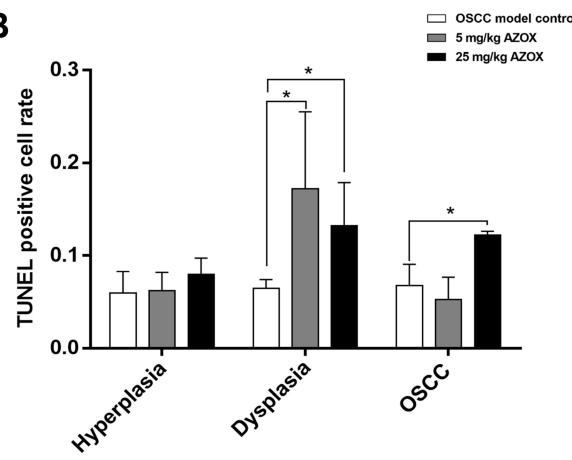

Figure 3 AZOX induces apoptosis in mouse tongue mucosa.

Notes: (A) TUNEL staining of apoptotic cells in mouse tongues in the OSCC model control, low-dose AZOX, and high-dose AZOX groups. (B) Apoptotic rates in mouse tongue tissues in each group (magnification $\times 200$ ). Data are presented as mean \pm SD. $* P<0.05$.

control group ( $P=0.009)$, as shown by Annexin V-fluorescein isothiocyanate/PI double staining. After treatment with $5 \mu \mathrm{g} /$ $\mathrm{mL}$ AZOX for $48 \mathrm{hrs}$, the early apoptotic rates of CAL27 and SCC15 cells increased significantly $(P=0.007$ and $P=0.046)$ (Figure 5A and B). These results suggest that AZOX induces the apoptosis of OSCC cells. CAL27 cells were shown to be more sensitive to AZOX than SCC15 cells.

\section{AZOX Induces Mitochondrial Dysfunctions in CAL27 Cells}

The activity of mitochondrial complex III was examined by measuring the change in the absorbance of cytochrome $\mathrm{c}$ at $550 \mathrm{~nm}$ for a certain period of time. The mitochondrial complex III activity value was $0.55 \pm 0.11$ in CAL27 cells treated with $5 \mu \mathrm{g} / \mathrm{mL}$ AZOX for $24 \mathrm{hrs}$, which was significantly lower than that in untreated CAL27 cells $(2.79 \pm 0.34)(P=0.000)$. This result suggests that AZOX significantly inhibits mitochondrial complex III activity in CAL27 cells (Figure 6A).

The effect of AZOX on the mitochondrial ROS level was assessed in CAL27 cells using the MitoSox fluorescent probe by flow cytometry. The red fluorescence of CAL27 cells was stronger after treatment with $5 \mu \mathrm{g} / \mathrm{mL}$ AZOX for $24 \mathrm{hrs}$ (Figure 6B). The red fluorescence intensity of CAL27 cells after treatment with $1.5,3$, and $6 \mu \mathrm{g} / \mathrm{mL}$ AZOX for $24 \mathrm{hrs}$ was higher than that of the control group (Figure 6C) $(P<0.01)$. Similarly, when the concentration of AZOX exceeded $3 \mu \mathrm{g} /$ $\mathrm{mL}$, mitochondrial ROS levels did not obviously change.

The effect of AZOX on intracellular ROS levels was investigated in CAL27 cells using a DHC probe and flow cytometry. The fluorescence of CAL27 cells was enhanced by treatment with $5 \mu \mathrm{g} / \mathrm{mL}$ AZOX for $24 \mathrm{hrs}$ (Figure 6D). Compared with the control, the fluorescence intensity of CAL27 cells was enhanced after treatment with 1.5, 3, and 6 $\mu \mathrm{g} / \mathrm{mL}$ AZOX for $24 \mathrm{hrs}$ (Figure $6 \mathrm{E})(P<0.01)$. However, when the AZOX concentration was greater than $3 \mu \mathrm{g} / \mathrm{mL}$, the fluorescence intensity did not continue to increase. These results indicate that a certain concentration of AZOX increases intracellular and mitochondrial ROS levels in CAL27 cells.

Intracellular MMP was detected in CAL27 cells using the JC-1 probe. In CAL27 cells treated with $5 \mu \mathrm{g} / \mathrm{mL}$ 
A

CAL27

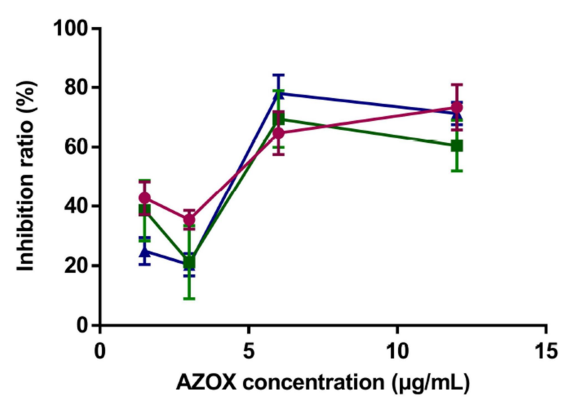

B
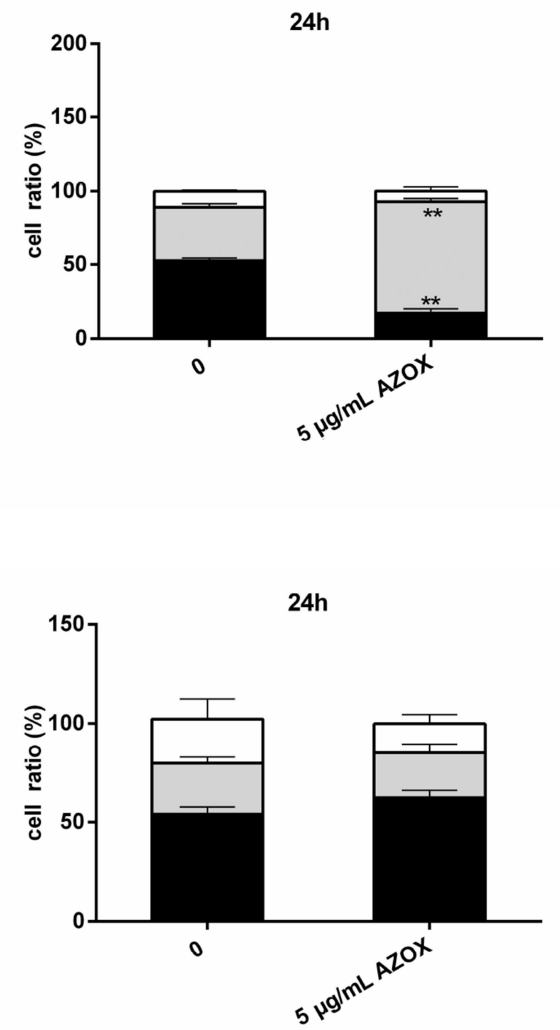

$\rightarrow 24 \mathrm{~h}$

$-48 \mathrm{~h}$

$+72 \mathrm{~h}$

- G0/G1
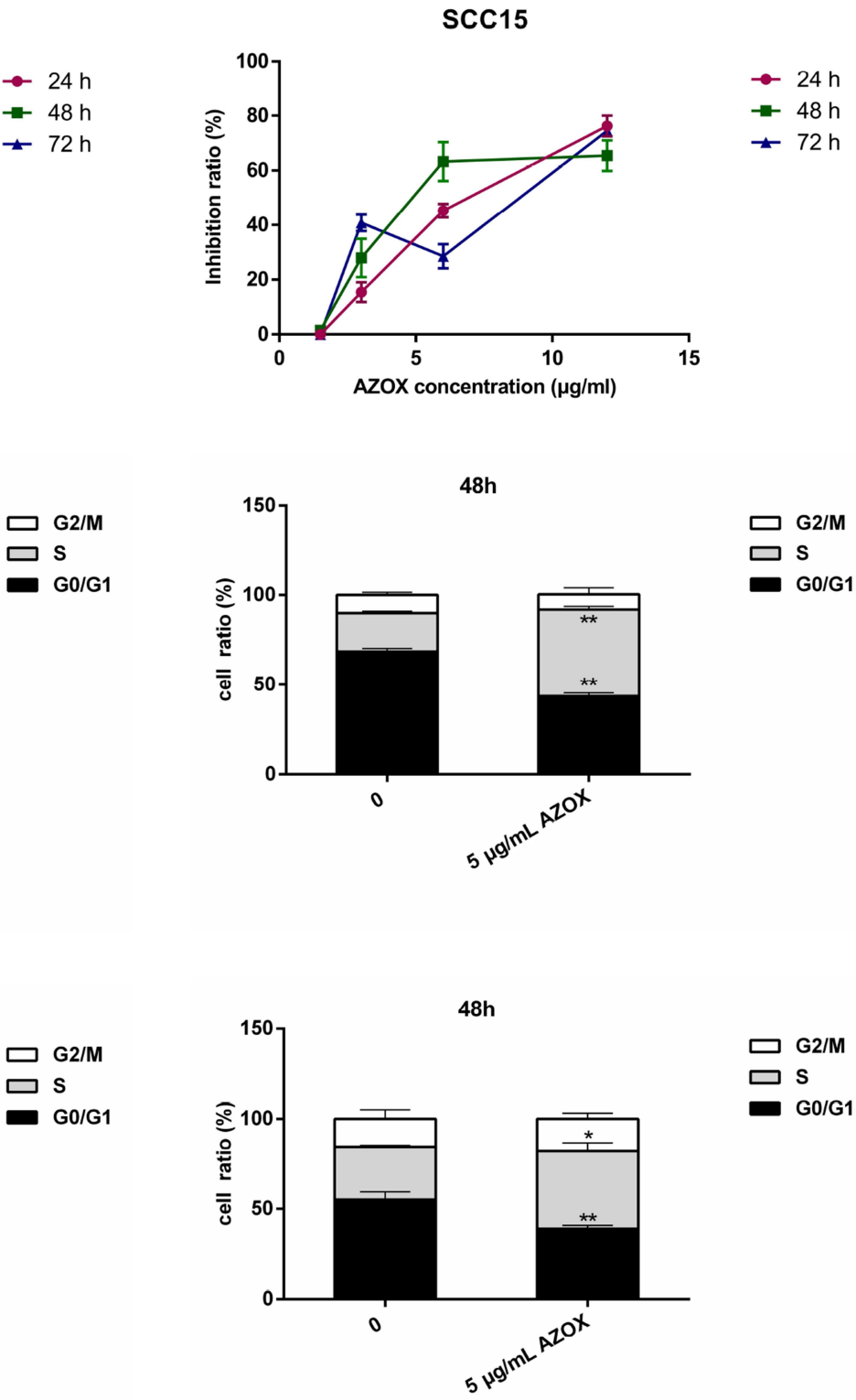

$\square$ G2/M

$\square \mathrm{s}$

G0/G1

Figure 4 AZOX inhibits cell viability and blocks the cell cycle in CAL27 and SCCI5 cells.

Notes: (A) Inhibition of OSCC cell viability by AZOX at different concentrations and time. (B) Effect of AZOX on CAL27 cell cycle. (C) Effect of AZOX on SCCI5 cell cycle. Data representing three independent experiments are shown as the mean $\pm S D$. $* P<0.05 ; * * P<0.01$.

AZOX for $24 \mathrm{hrs,}$, red fluorescence was weaker, whereas green fluorescence was stronger in these cells than that in the model control group (Figure 6F). The ratio of JC-1 monomers in CAL27 cells increased, indicating a decrease in intracellular MMP. The ratio of red to green fluorescence intensity gradually decreased in CAL27 cells treated with $1.5,3$, and $6 \mu \mathrm{g} / \mathrm{mL}$ AZOX for $24 \mathrm{hrs}$, and the difference between AZOX treatment and control was statistically significant $(P<0.01)$ (Figure 6G). These results suggest that AZOX induces cell apoptosis by decreasing MMP in CAL27 cells.

\section{Discussion}

As a monomeric compound, AZOX has a well-defined composition: molecular formula, C22H17N3O5; molecular weight, 403.3875 M; and Chemical Abstracts Service, No. 131,860-3-8. Toxicological experiments have shown that AZOX has low toxicity, with LD50 values of 

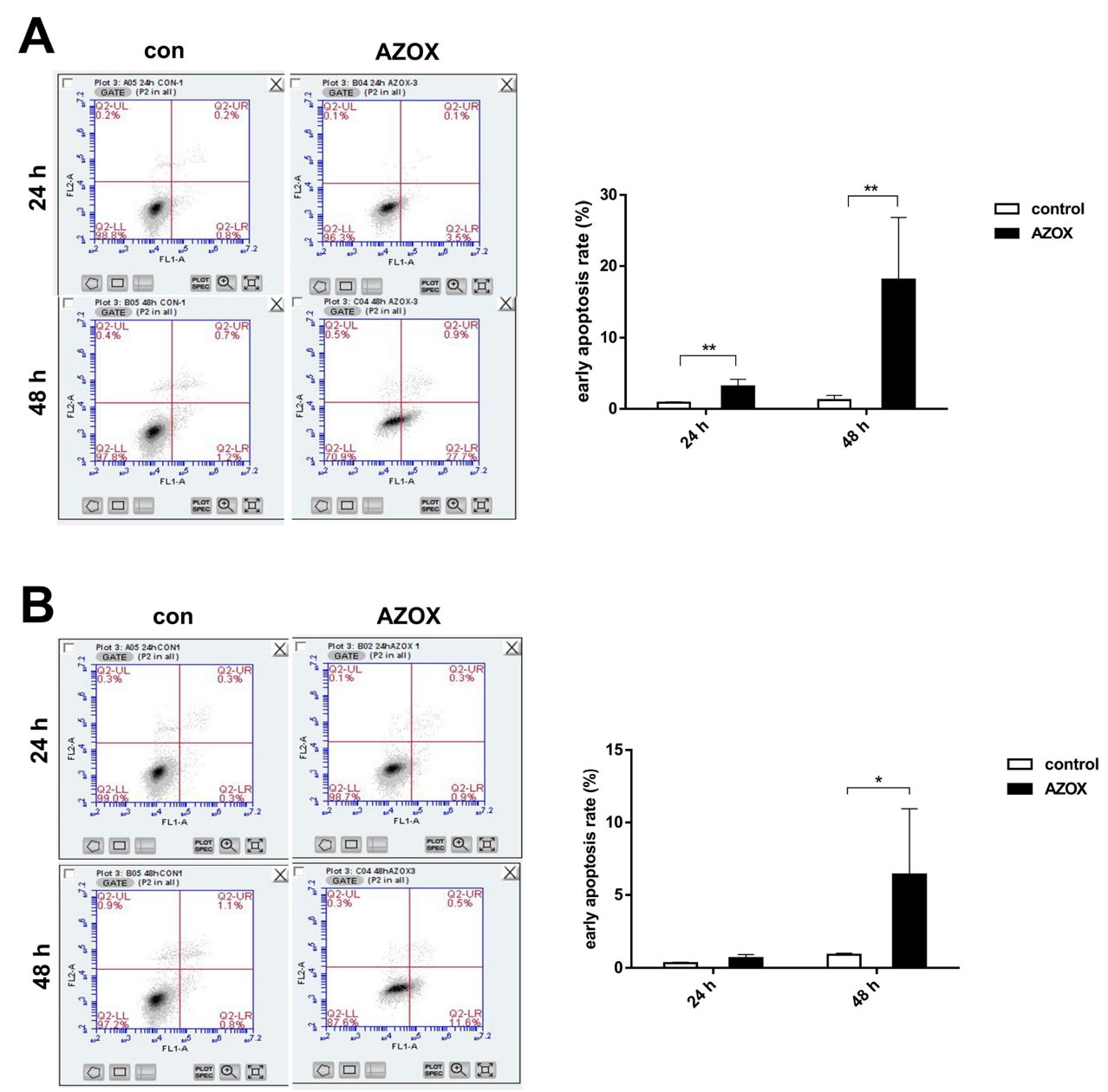

Figure 5 AZOX induces apoptosis in CAL27 and SCCI5 cells.

Notes: (A) Apoptosis of CAL27 cells after treatment with $5 \mu \mathrm{g} / \mathrm{mL}$ AZOX for 24 and $48 \mathrm{hrs}$. (B) Apoptosis of SCCI5 cells after treatment with $5 \mu \mathrm{g} / \mathrm{mL}$ AZOX for 24 and $48 \mathrm{hrs}$. Data are presented as mean \pm SD. $* P<0.05$; $* * P<0.01$.

$>5000 \mathrm{mg} / \mathrm{kg}$ via oral administration and $>5000 \mathrm{mg} / \mathrm{kg}$ via acute percutaneous injection in female and male rats. AZOX does not cause chromosomal aberrations in human peripheral blood lymphocytes, and no mutagenic, teratogenic, and carcinogenic effects of AZOX have been reported. ${ }^{3}$ In this study, we also showed that AZOX had high biosafety by calculating the main organ coefficients in each group of mice.

A murine 4NQO-induced model can well mimic human oral carcinogenesis in terms of its morphological, histopathological, and molecular features. $^{9-11}$ To examine the preventive effects of AZOX on oral carcinogenesis, 4NQO was used to induce mouse tongue cancer in this study. From the 10th week, in which no gross precancerous or cancerous lesions were observed, high- $(25 \mathrm{mg} / \mathrm{kg})$ and low-dose $(5 \mathrm{mg} / \mathrm{kg})$ AZOX were intragastrically administered every other day. Compared with the model control group, the high-dose AZOX group showed a significantly reduced incidence of tongue cancer from $70 \%$ to $20 \%$ and attenuated tongue lesion severity. Most mouse tongue lesions were arrested at the epithelial-moderate dysplastic stage, indicating that high-dose AZOX exerted a significant inhibitory effect on the development of oral cancer. High-dose AZOX significantly inhibited cell viability and induced apoptosis in the epithelial dysplasia and OSCC lesions in mouse tongue mucosa. In vitro, AZOX significantly inhibited cell viability and induced apoptosis in CAL27 and SCC15 cells. The cell cycle was arrested in the $S$ phase. A recent study has shown that AZOX inhibits the growth of human esophageal squamous cell carcinoma KYSE-150 cells in transplanted tumors by inhibiting cell proliferation and inducing apoptosis. ${ }^{7}$ These results indicated that AZOX induced apoptosis, inhibited cell viability, and ultimately inhibited the development of oral cancer. However, the mechanism of the inhibitory effects of AZOX on oral cancer development remains to be elucidated. 
A

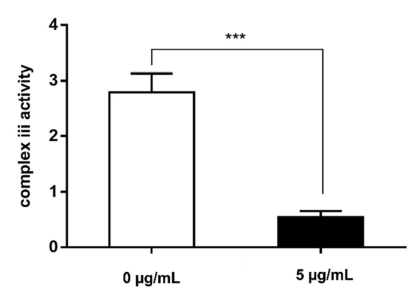

B

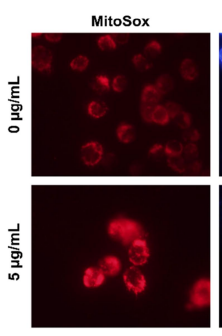

C
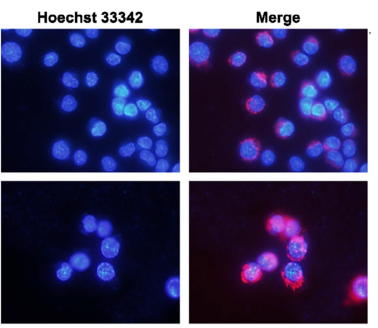
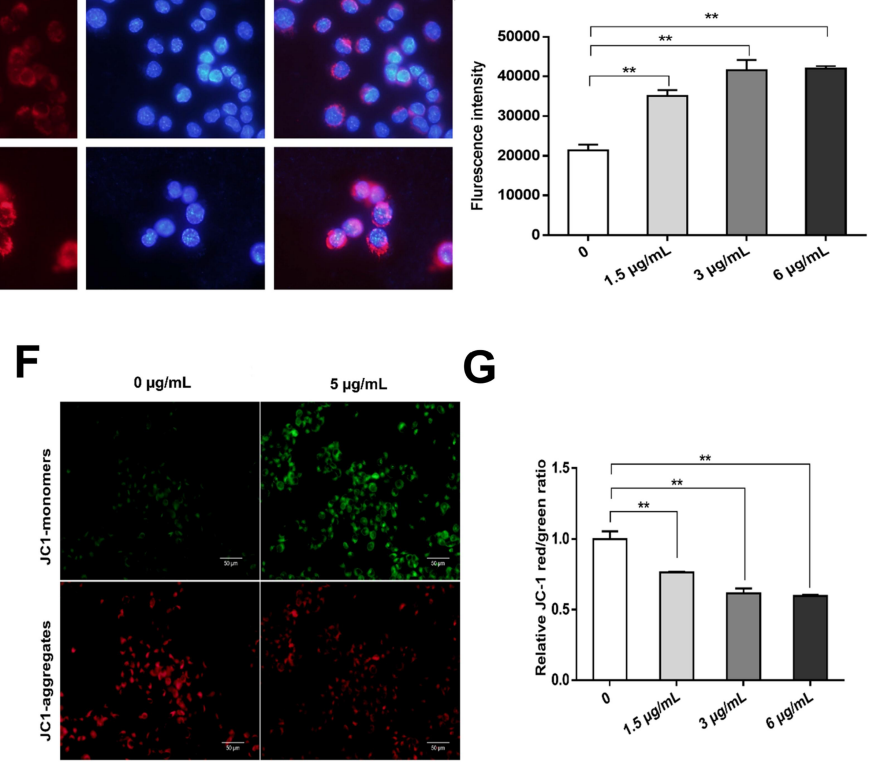

Figure 6 AZOX induces mitochondrial dysfunctions in CAL27 cells.

Notes: (A) Changes in mitochondrial complex III activity in CAL27 cells treated with $5 \mu \mathrm{g} / \mathrm{mL}$ AZOX for 24 hrs. (B) Effect of AZOX on mitochondrial ROS levels in CAL27 cells treated with $5 \mu \mathrm{g} / \mathrm{mL}$ AZOX for $24 \mathrm{hrs}$ (magnification $\times 200$ ). (C) Mitochondrial ROS level of CAL27 cells after treatment with 0 , I.5, 3 and $6 \mu \mathrm{g} / \mathrm{mL}$ AZOX. (D) Effect of AZOX on the ROS level of CAL27 cells treated with $5 \mu \mathrm{g} / \mathrm{mL}$ AZOX for $24 \mathrm{hrs}$ (magnification $\times 200$ ). (E) ROS level of CAL27 cells after treatment with 0 , I.5, 3 , and 6 $\mu \mathrm{g} / \mathrm{mL}$ AZOX for $24 \mathrm{hrs}$. (F) Effect of AZOX on the MMP of CAL27 cells treated with $5 \mu \mathrm{g} / \mathrm{mL}$ AZOX for 24 hrs (magnification $\times 200$ ). (G) The ratio of red to green fluorescence intensity of CAL27 cells treated with $0,1.5,3$, and $6 \mu \mathrm{g} / \mathrm{mL}$ AZOX for $24 \mathrm{hrs}$. Data are presented as mean \pm SD. $* * P<0.01$; $* * * P=0.000$.

Abbreviations: ROS, reactive oxygen species; MMP, mitochondrial membrane potential.

Studies have reported that AZOX is a mitochondrial complex III inhibitor that tightly binds to the Qo site of the cytochrome bcl complex (complex III) in fungi. ${ }^{12}$ Mitochondrial complex III is an important transmembrane protein complex in the mitochondrial respiratory chain. ${ }^{13}$ It has two functional binding sites for quinone: site Qo, which is located on the side of the membrane gap, and site Qi, which is located at one side of the substrate. ROS is mainly generated from the Qo site, in which molecular oxygen is changed into its superoxide anion. Our in vivo and in vitro results showed that AZOX significantly induced apoptosis in OSCC. So we deduced that AZOX may induce apoptosis by targeting mitochondrial complex III and affecting mitochondrial functions in oral cancer. The results of this study showed that AZOX significantly inhibited the activity of mitochondrial complex III, increased the levels of both mitochondrial and intracellular ROS in CAL27 cells. Our results are consistent with those of previous studies, in which inhibition of mitochondrial complex III activity accelerated ROS production. ${ }^{14}$ In addition, Guo et al found that AZOX suppresses the activity of mitochondrial complex III in a dose-dependent manner and regulates glucose and lipid metabolism in high-fat diet-fed mice. ${ }^{15}$ In zebrafish, exposed to $100 \mu \mathrm{g} /$ L AZOX for 7, 14, and 21 days, the ROS level in the liver significantly increases in a dose-dependent manner. ${ }^{16}$ The present results suggested that AZOX induces ROS accumulation by inhibiting the activity of mitochondrial complex III in CAL27 cells. However, other researchers also observed that AZOX prevented hydroquinone from transporting electrons to the $\mathrm{Fe}-\mathrm{S}$ center and electron transfer chain in the cytochrome bc1 complex and inhibited ROS production. ${ }^{17}$ Moreover, intracellular ROS levels significantly decreased in 3T3-L1 and HepG2 cells treated with $5 \mathrm{mM}$ AZOX for 30 mins. ${ }^{18}$ Therefore, differences in the effects of AZOX on ROS levels need to be further investigated.

Research revealed that ROS accumulation participates in apoptotic signaling by inducing MMP loss and inhibiting $\mathrm{Bcl}-2$ and cytochrome $c$ extrusion from the mitochondria. ${ }^{19}$ MMP decrease can result in the release of cytochrome $c$ and AIF to facilitate effector caspase cascade activation, which involves caspase- 9 and $-3 .^{20}$ A previous research showed that changes in MMP precede nuclear destruction in apoptosis, and MMP decrease suggests early apoptosis. ${ }^{21}$ In the present study, MMP was 
significantly reduced in CAL27 cells treated with AZOX, suggesting that AZOX induced early apoptosis. MMP decrease can produce and release of ROS into the cytosol, which further induces apoptosis. These data suggested that the inhibitory action of AZOX on the activity of mitochondrial complex III, which resulted in mitochondrial respiratory chain dysfunction, induced an increase in mitochondrial ROS level. Excessive ROS led to decreased MMP and inducing apoptosis in oral cancer cells. Further studies are needed on the mechanism of AZOX in oral cancer to identify its potential drug targets.

\section{Conclusion}

We, for the first time, found AZOX to exert significant inhibitory effects on oral carcinogenesis by specifically targeting mitochondrial complex III, leading to ROS accumulation, MMP decrease and apoptosis. AZOX may thus be a candidate agent for the prevention and treatment of OSCC.

\section{Data Sharing Statement}

The datasets used and/or analyzed during the current study are available from the corresponding author on reasonable request.

\section{Ethics Approval}

The experiments were conducted in accordance with the UK Animals (Scientific Procedures) Act and the Beijing Municipality on the Review of Welfare and Ethics of Laboratory Animals guidelines approved by the Beijing Municipality Administration Office of Laboratory Animals (BAOLA). The study was approved by the Animal Ethical and Welfare Committee of Beijing Stomatological Hospital (approval No. KQYY-201,804-010).

\section{Acknowledgments}

We would like to thank all the individuals who helped with this project.

\section{Funding}

This study was funded by Natural Science Foundation of Beijing Municipality (Grant No: 7192075).

\section{Disclosure}

Dr Hui Chen and Dr Xiaofei Tang report a patent issued: Effects of Azoxystrobin on oral cancer and precancerous lesions. The authors declare that they have no other potential conflicts of interest for this work.

\section{References}

1. Marur S, Forastiere AA. Head and neck squamous cell carcinoma: update on epidemiology, diagnosis, and treatment. Mayo Clin Proc. 2016;91(3):386-396. doi:10.1016/j.mayocp.2015.12.017

2. Koyfman SA, Ismaila N, Crook D, et al. Management of the neck in squamous cell carcinoma of the oral cavity and oropharynx: ASCO clinical practice guideline. J Clin Oncol. 2019;37(20):1753-1774. doi:10.1200/JCO.18.01921

3. Iqbal Z, Han L-C, Soares-Sello AM, et al. Investigations into the biosynthesis of the antifungal strobilurins. Org Biomol Chem. 2018;16(30):5524-5532. doi:10.1039/C8OB00608C

4. Rodrigues ET, Lopes I, Pardal MÂ. Occurrence, fate and effects of azoxystrobin in aquatic ecosystems: a review. Environ Int. 2013;53:18-28. doi:10.1016/j.envint.2012.12.005

5. Wiggins TE, Jager BJ. Mode of action of the new methoxyacrylate antifungal agent ICIA5504. Biochem Soc Trans. 1994;22(1):68S. doi:10.1042/bst022068s

6. Cao F, Wu P, Huang L, et al. Short-term developmental effects and potential mechanisms of azoxystrobin in larval and adult zebrafish (Danio rerio). Aquat Toxicol. 2018;198:129-140. doi:10.1016/j. aquatox.2018.02.023

7. Shi XK, Bian XB, Huang T, et al. Azoxystrobin induces apoptosis of human esophageal squamous cell carcinoma KYSE-150 cells through triggering of the mitochondrial pathway. Front Pharmacol. 2017;8:277. doi:10.3389/fphar.2017.00277

8. Boivin GP, Bottomley MA, Schiml PA, Goss L, Grobe N. Physiologic, behavioral, and histologic responses to various euthanasia methods in C57BL/6NTac male mice. J Am Assoc Lab Anim Sci. 2017;56(1):69-78.

9. Tang XH, Knudsen B, Bemis D, Tickoo S, Gudas LJ. Oral cavity and esophageal carcinogenesis modeled in carcinogen-treated mice. Clin Cancer Res. 2004;10(1):301-313. doi:10.1158/1078-0432.CCR-0999-3

10. Liu M, Su Y, Lan AX, Zhang XY, Sun Z. Expression of KLF5 in 4NQO-induced mouse tongue carcinogenesis. Beijing J Stomatol. 2014;3:125-128.

11. Kanojia D, Vaidya MM. 4-nitroquinoline-1-oxide induced experimental oral carcinogenesis. Oral Oncol. 2006;42(7):655-667. doi:10.1016/j.oraloncology.2005.10.013

12. Esser L, Quinn B, Li YF, et al. Crystallographic studies of quinol oxidation site inhibitors: a modified classification of inhibitors for the cytochrome bc(1) complex. J Mol Biol. 2004;341(1):281-302. doi:10.1016/j.jmb.2004.05.065

13. Enns L, Ladiges W. Mitochondrial redox signaling and cancer invasiveness. $J$ Bioenerg Biomembr. 2012;44(6):635-638. doi:10.1007/s10863-012-9467-7

14. Korge P, John SA, Calmettes G, Weiss JN. Reactive oxygen species production induced by pore opening in cardiac mitochondria: the role of complex II. J Biol Chem. 2017;292(24):9896-9905. doi:10.1074/ jbc.M116.768325

15. Gao AH, Fu YY, Zhang KZ, et al. Azoxystrobin, a mitochondrial complex III Qo site inhibitor, exerts beneficial metabolic effects in vivo and in vitro. Biochim Biophys Acta. 2014;1840 (7):2212-2221. doi:10.1016/j.bbagen.2014.04.002

16. Han Y, Liu T, Wang J, et al. Genotoxicity and oxidative stress induced by the fungicide azoxystrobin in zebrafish (Danio rerio) livers. Pestic Biochem Physiol. 2016;133:13-19. doi:10.1016/j. pestbp.2016.03.011

17. Boudina A, Emmelin C, Baaliouamer A, Paisse O, Chovelon JM. Photochemical transformation of azoxystrobin in aqueous solutions. Chemosphere. 2007;68(7):1280-1288. doi:10.1016/j.chemosphere. 2007.01.051

18. Bhattacharya K, Bag AK, Tripathi R, et al. Mahanine, a novel mitochondrial complex-III inhibitor induces G0/G1 arrest through redox alteration-mediated DNA damage response and regresses glioblastoma multiforme. Am J Cancer Res. 2014;4(6):629-647. 
19. Narrima P, Paydar M, Looi CY, et al. Persea declinata (B1.) kosterm bark crude extract induces apoptosis in MCF-7 cells via G0/G1 cell cycle arrest, $\mathrm{Bcl}-2 / \mathrm{Bax} / \mathrm{Bcl}-\mathrm{xl}$ signaling pathways, and ROS generation. Evid Based Complement Alternat Med. 2014;2014:248103. doi:10.1155/ 2014/248103

20. Madungwe NB, Feng Y, Lie M, et al. Mitochondrial inner membrane protein (mitofilin) knockdown induces cell death by apoptosis via an AIF-PARP-dependent mechanism and cell cycle arrest. Am J Physiol Cell Physiol. 2018;315(1):C28-C43. doi:10.1152/ajpcell.00230.2017
21. Huang ML-H, Chiang S, Kalinowski DS, et al. The role of the antioxidant response in mitochondrial dysfunction in degenerative diseases: cross-talk between antioxidant defense, autophagy, and apoptosis. Oxid Med Cell Longev. 2019;2019:6392763. doi:10.1155/ 2019/6392763

\section{Publish your work in this journal}

Cancer Management and Research is an international, peer-reviewed open access journal focusing on cancer research and the optimal use of preventative and integrated treatment interventions to achieve improved outcomes, enhanced survival and quality of life for the cancer patient.
The manuscript management system is completely online and includes a very quick and fair peer-review system, which is all easy to use. Visit http://www.dovepress.com/testimonials.php to read real quotes from published authors. 